\title{
SIMPLE MAXIMAL QUOTIENT RINGS
}

\author{
ROBERT A. RUBIN
}

\begin{abstract}
In this paper we consider the question of when a ring $\Lambda$ has a simple maximal left ring of quotients. In the first section we determine two necessary conditions; viz. that $\Lambda$ be left nonsingular, and when $I$ and $J$ are nonzero ideals of $\Lambda$ with $I \cap J=0$, then $I+J$ is not left essential in $\Lambda$. In the second section we show that these conditions are also sufficient when $\Lambda$ is of finite left Goldie dimension. In addition, for a left nonsingular ring of finite left Goldie dimension, we determine the ideal structure of the maximal left ring of quotients.
\end{abstract}

Note. Any unadorned terms, e.g., nonsingular, finite dimensional, maximal ring of quotients, are intended to hold on the left.

1. Necessary conditions. Various conditions sufficient to guarantee a simple maximal ring of quotients are known, the most general of these being that the ring be absolutely torsion-free [2, Theorem 2.1]. Since the maximal ring of quotients of the $n \times n$ triangular matrix ring over a field is simple, the preceding condition is certainly not necessary. In this section we determine certain necessary conditions for a ring to have a simple maximal ring of quotients. The first such condition is rather familiar, namely that the ring be (left) nonsingular.

Proposition 1.1. Let $\Lambda$ be a ring with maximal ring of quotients $Q$. If $Q$ is simple, then $\Lambda$ is (left) nonsingular.

Proof. If $\mathfrak{A}$ is an essential left ideal of $\Lambda$, then $Q \mathfrak{U}$ is an essential left ideal of $Q$, for if $0 \neq q \in Q$, then there exists $b \in \Lambda$ such that $0 \neq b q \in \Lambda$. Then there is $r \in \Lambda$ such that $0 \neq(r b) q=r(b q) \in \mathfrak{U} \subseteq Q \mathfrak{A}$. Now if $Q$ is a simple ring, then $Q$ is nonsingular (as a $Q$-module), whence $\Lambda$ must also be nonsingular.

With Proposition 1.1 in hand we can obtain a formally weaker condition equivalent to having a simple maximal ring of quotients.

Proposition 1.2. Let $\Lambda$ be a ring with maximal ring of quotients $Q$. Then $Q$ is simple if and only if $Q$ is an absolutely torsion-free ring; i.e. if for every kernel functor (left exact preradical) $\delta$ of $Q$, either $\delta(Q)=0$ or $\delta(Q)=Q$.

Proof. Since any simple ring is absolutely torsion-free, one implication is immediate. Now assume that $Q$ is absolutely torsion-free. Then $Q$ is nonsin-

Received by the editors May 7, 1974 and, in revised form, December 6, 1974.

AMS (MOS) subject classifications (1970). Primary 16A08, 16A40.

Key words and phrases. Simple ring, nonsingular ring, Goldie dimension, maximal ring of quotients, semisimple ring, self-injective ring. 
gular, whence as in the proof of Proposition 1.1, $\Lambda$ is also nonsingular. Therefore $Q$ is (left) self-injective, and so the proof of Theorem 2.1 of [2] applies. Thus $Q$ is simple.

We next obtain a necessary condition in terms of the ideal structure of $\Lambda$.

THEOREM 1.3. Let $\Lambda$ be a ring with maximal ring of quotients $Q$. Suppose that $Q$ is simple. Then for any nonzero ideals $I, J$ of $\Lambda$, if $I \cap J=0$, then $I+J$ is not essential as a left ideal of $\Lambda$.

Proof. Suppose that $I$ and $J$ are nonzero ideals with $I \cap J=0$ and $I+J$ essential. We will show that $I Q J=0$, whence it follows that $Q I Q$ is a proper nonzero ideal of $Q$, a contradiction. So let $a \in I, b \in J, q \in Q$, and suppose that $a q b \neq 0$. Since $I+J$ is essential and $\Lambda$ is nonsingular (Proposition 1.1), there is $r \in \Lambda$ such that $r a q b \neq 0$ and $r a q \in I+J$. Thus $0 \neq r a q=x+y$, for some $x \in I, y \in J$. Now $I($ raq $-x)=I y=0$, and $J(r a q-x)=0$, since $r a, x \in I$. Thus $(I+J)(r a q-x)=0$, and since $\Lambda$ is nonsingular, $r a q \in I$. But then $r a q b \in I J=0$, a contradiction. Thus $I Q J=0$, and the theorem is proved.

COROLlaRy 1.4. If $\Lambda$ is a semiprime ring with simple maximal ring of quotients, then every nonzero ideal of $\Lambda$ is essential as a left ideal.

Proof. Let $I$ be a nonzero ideal of $\Lambda$. If $I$ is not left essential in $\Lambda$, then there is a left ideal $\mathfrak{A} \neq 0$ of $\Lambda$ such that $I \cap \mathfrak{A}=0$ and $I+\mathfrak{U}$ is left essential. But $\mathfrak{A} \Lambda \cap I$ is nilpotent and, hence, zero. So we have $I \cap \mathfrak{A} \Lambda$ $=0$ and $I+\mathfrak{A} \Lambda$ left essential, contradicting Theorem 1.3. Thus $I$ is essential.

2. The finite-dimensional case. In this section we show that for rings of finite left Goldie dimension (rings with no infinite collection of nonzero left ideals whose sum is direct) the converse of Theorem 1.3 holds. In addition, for nonsingular finite-dimensional rings, we ascertain the ideal structure of the maximal ring of quotients.

Proposition 2.1. Let $\Lambda$ be a (left) finite-dimensional ring with maximal ring of quotients $Q$. If $Q$ is simple, then $Q$ is semisimple (with d.c.c.).

Proof. By Proposition 1.1, $\Lambda$ is nonsingular. Hence, Theorem 1.6 of [3] applies, yielding the result.

REMARK. The results of this paper can be used to strengthen Theorem 1.6 of [3] a bit. Namely, we can easily show that a ring $\Lambda$ has a semisimple maximal ring of quotients if and only if $\Lambda$ is nonsingular and finite dimensional. For if $\Lambda$ has a semisimple maximal ring of quotients $Q$, then $Q$ is nonsingular, whence $\Lambda$ is nonsingular as well. Then the theorem we are discussing applies, from which we conclude that $\Lambda$ is finite dimensional.

THEOREM 2.2. Let $\Lambda$ be a (left) finite-dimensional (left) nonsingular ring with maximal ring of quotients $Q$. Then the following are equivalent:

(i) $Q$ is a simple ring;

(ii) for any nonzero ideals $I$ and $J$ of $\Lambda$, if $I \cap J=0$, then $I+J$ is not left essential in $\Lambda$.

Proof. (i) $\Rightarrow$ (ii). This is just Theorem 1.3. 
(ii) $\Rightarrow$ (i). Let $X$ be a nonzero ideal of $Q$. Since $Q$ is semisimple, $X=Q E$, $e$ a nonzero central idempotent of $Q$. Let $f=1-e$. Then $f$ is also a central idempotent of $Q$, and $\{r \in \Lambda \mid r e \in \Lambda\}=\{r \in \Lambda \mid r f \in \Lambda\}$. This set, which we denote $D$, is an ideal, left essential in $\Lambda$. Let $I=D e$ and $J=D f$. Then $I$ and $J$ are ideals of $\Lambda$ with $I \cap J=0$. Furthermore $(D e) e=D e \subseteq \Lambda$, and $(D f) f=D f \subseteq \Lambda$. Thus $I+J \subseteq D$. Finally if $d \in D$, then $d=d e+d f$ $\in I+J$. Thus $I+J$ is left essential in $\Lambda$. So by (ii), $J=0$, whence $f=0$ and $X=Q$. Thus $Q$ is a simple ring.

REMARK. While condition (ii) of the preceding theorem may not be equivalent to having a simple maximal ring of quotients in general, an examination of the proof of the theorem reveals that the condition is equivalent to the nonexistence of central idempotents $\neq 0,1$ in $Q$.

We now determine the ideal structure of the maximal ring of quotients of a finite-dimensional nonsingular ring. So let $\Lambda$ be such a ring, and let $Q$ be its maximal ring of quotients. If $I$ is an ideal of $\Lambda$, let $A(I)=\{r \in \Lambda \mid I r=0\}$. Then $A(I)$ is an ideal of $\Lambda$.

Let $X$ be an ideal of $Q$. Since $Q$ is semisimple [3, Theorem 1.6], $X=Q e$ for some central idempotent $e$ of $Q$. Let $f=1-e$, and $D=\{r \in \Lambda \mid r e \in \Lambda\}$ $=\{r \in \Lambda \mid r f \in \Lambda\}$. Then, as in the proof of Theorem 2.2, $D e+D f=D$, which is left essential in $\Lambda$. Note that $D e=Q e \cap \Lambda=X \cap \Lambda$, for if $q e \in \Lambda$, then $(q e) e \in \Lambda$, whence $q e \in D$, and so $q e=q e^{2} \in D e$. The other inclusion is immediate. Similarly $D f=Q f \cap \Lambda$. Now $(D e)(D f)=0$, while if $(D e) r=0$, then $D(e r)=0$, whence the nonsingularity of $\Lambda$ implies $e r=0$. Thus $r \in Q f \cap \Lambda$, and so $D f=A(D e)$. Similarly, $D e=A(D f)$. Thus if $X$ is an ideal of $Q$, and if $I=X \cap \Lambda$, then $I=A(A(I))$.

Conversely, suppose that $\mathrm{I}$ is an ideal of $\Lambda$ such that $I=A(A(I))$. Then $I$ is closed as a left ideal; i.e. I has no proper left essential extensions in $\Lambda$. For if $\mathfrak{B}$ is a proper essential extension of $I$, then since $I=A(A(I))$, there is $x \in \mathfrak{B}$ and $a \in A(I)$ such that $a x \neq 0$. Now for some essential left ideal $\mathfrak{D}$, Dax $\subseteq I$. But Dax $\subseteq A(I)$ as well, and so Dax $\subseteq I \cap A(I)$. Now $I \cap A(I)$ $=A(A(I)) \cap A(I)$ is zero since it is annihilated by the left essential ideal $I+A(I)$. Thus $\mathfrak{D} a x=0$, contradicting the nonsingularity of $\Lambda$. Next, observe that $I \subseteq I Q \cap \Lambda$. Furthermore, if $x \in I$ and $q \in Q$ are such that $0 \neq x q$ $\in \Lambda$, then there is an essential left ideal $\mathfrak{D}$ of $\Lambda$ with $\mathfrak{D} \subseteq I+A(I)$. If $d \in \mathfrak{D}$ is such that $d x q \neq 0$, then $d=d_{1}+d_{2}$, with $d_{1} \in I$ and $d_{2} \in A(I)$, whence $d x q=d_{1} x q+d_{2} x q=d_{1} x q \in I$. It now follows that $I$ is left essential in $I Q \cap \Lambda$, whence $I=I Q \cap \Lambda$. Therefore $Q I=Q(I Q \cap \Lambda)$. Now the conditions of Theorem 1.6 of [3], which hold in the situation under consideration, are equivalent to $Q$ being a right flat $\Lambda$-module, and the inclusion of $\Lambda$ in $Q$ being an epimorphism of rings [4, Theorem 13.10]. Therefore Lemma 1.10 of [1] applies, and so $Q I=Q(I Q \cap \Lambda)=Q I Q \cap Q \Lambda=Q I Q$. Thus $Q I$ is an ideal of $Q$.

We summarize the preceding in the following:

THEOREM 2.3. Let $\Lambda$ be a nonsingular, finite-dimensional ring, and let $Q$ be its maximal ring of quotients. For any ideal I of $\Lambda$, let $A(I)=\{r \in \Lambda \mid \operatorname{Ir}=0\}$. Then there is a one-to-one correspondence between ideals $X$ of $Q$ and ideals $I$ of $\Lambda$ satisfying $I=A(A(I))$. The correspondence is given by $X \mapsto X \cap \Lambda$, and $I$ $\mapsto Q I$. 


\section{REFERENCES}

1. V. C. Cateforis, Flat regular quotient rings, Trans. Amer. Math. Soc. 138 (1969), 241-249. MR $39 \# 259$.

2. R. A. Rubin, Absolutely torsion-free rings, Pacific J. Math. 46 (1973).

3. F. L. Sandomierski, Semisimple maximal quotient rings, Trans. Amer. Math. Soc. 128 (1967), 112-120. MR 35 \#5473.

4. B. Stenström, Rings and modules of quotients, Lecture Notes in Math., vol. 237, SpringerVerlag, Berlin and New York, 1971. MR 48 \#010.

Department of Mathematics and Statistics, Pahlavi University, Shiraz, Iran 